\title{
Sputum microbiota in adults with CF associates with response to inhaled tobramycin
}

\author{
Alya Heirali, ${ }^{1}$ Christina Thornton, ${ }^{2}$ Nicole Acosta, ${ }^{1}$ Ranjani Somayaji, ${ }^{2}$ \\ Isabelle Laforest Lapointe, ${ }^{3}$ Douglas Storey, ${ }^{4}$ Harvey Rabin, ${ }^{2}$ Barbara Waddell, ${ }^{1}$ \\ Laura Rossi, ${ }^{5}$ Marie Claire Arrieta, ${ }^{6,7}$ Michael Surette, ${ }^{8}$ Michael D Parkins ${ }^{2}$
}

\begin{abstract}
- Additional material is published online only. To view, please visit the journal online (http://dx.doi.org/10.1136/ thoraxjn-2019-214191)
\end{abstract}

${ }^{1}$ Microbiology, Immunology and Infectious Diseases, University of Calgary, Calgary, Alberta, Canada

${ }^{2}$ Medicine, University of Calgary, Calgary, Alberta, Canada Département de Biologie, Universite de Sherbrooke, Sherbrooke, Quebec, Canada ${ }^{4}$ Biological Sciences, University of Calgary, Calgary, Alberta,

Canada

${ }^{5}$ Microbiology, McMaster University, Hamilton, Ontario, Canada

${ }^{6}$ Pediatrics, Calgary, Alberta, Canada

${ }^{7}$ Physiology \& Pharmacology, University of Calgary, Calgary, Alberta, Canada

${ }^{8}$ Medicine, McMaster University, Hamilton, Ontario, Canada

\section{Correspondence to} Dr Michael D Parkins, Medicine, University of Calgary, Calgary, AB T2N 1N4, Canada; mdparkin@ucalgary.ca

Received 10 October 2019 Revised 3 August 2020 Accepted 9 August 2020 Published Online First 2 November 2020

\section{Linked}

- http://dx.doi.org/10.1136/ thoraxjnl-2020-216005

\section{Check for updates}

(C) Author(s) (or their employer(s)) 2020. No commercial re-use. See rights and permissions. Published by BMJ.

To cite: Heirali A, Thornton C Acosta N, et al. Thorax

2020;75:1058-1064.

\section{ABSTRACT}

Background Inhaled tobramycin powder/solution (TIP/S) use has resulted in improved clinical outcomes in patients with cystic fibrosis (CF) with chronic Pseudomonas aeruginosa. However, TIP/S effect on the CF sputum microbiome has not been explored. We hypothesised that TIP/S has additional 'off-target' effects beyond merely $P$. aeruginosa and that baseline microbiome prior to initiation of therapy is associated with subsequent patient response.

Methods We drew sputum samples from a prospectively collected biobank. Patients were included if they had one sputum sample in the 18 months before and after TIP/S. Bacterial 16S rRNA gene profiling was used to characterise the sputum microbiome.

Results Forty-one patients met our inclusion criteria and 151 sputum samples were assessed. At baseline, median age was 30.4 years (IQR 24.2-35.2) and forced expiratory volume in $1\left(\mathrm{FEV}_{1}\right)$ second was $57 \%$ predicted (IQR 44-74). Nineteen patients were defined a priori as responders having no net decrease in $\mathrm{FEV}_{\text {, }}$ in the year following TIP/S. No significant changes were observed in key microbiome metrics of alpha (within-sample) or beta (between-sample) diversity for samples collected before and after TIP/S. However, significant betadiversity (Bray-Curtis) differences were noted at baseline between patients based on response status. Notably, responders were observed to have a higher abundance of Staphylococcus in pretherapy baseline samples.

Conclusions Our longitudinal study demonstrates that the sputum microbiome of patients with CF is relatively stable following inhaled tobramycin over many months. Intriguingly, our findings suggest that baseline microbiome may associate with patient response to TIP/S - suggesting the sputum microbiome could be used to personalise therapy.

\section{INTRODUCTION}

Cystic fibrosis (CF) is caused by mutations in the CF transmembrane conductance regulatory gene ultimately resulting in defective ion transport across epithelial cells. This leads to the buildup of mucus in the respiratory tract-serving as an ideal environment for chronic bacterial infections. Pseudomonas aeruginosa (PA), the archetypal CF pathogen, infects up to $60 \%$ of patients with CF and chronic infection is associated with worse outcomes. ${ }^{1}$ Accordingly, clinicians prescribe inhaled antipseudomonal antibiotics to manage these chronic infections. ${ }^{2}$ Indeed, inhaled antibiotics enable supratherapeutic drug

\section{Key messages}

What is the key question?

- We sought to determine if baseline microbial community composition of sputum in adults with cystic fibrosis initiating inhaled tobramycin associated with subsequent clinical outcome.

What is the bottom line?

- Microbial communities in expectorated sputum were different between those who exhibited a forced expiratory volume in $1 \mathrm{~s}$ response to tobramycin and those who did not; in particular responders had communities rich in Staphylococcus.

Why read on?

- Microbial community analysis in the context of inhaled antibiotics may provide unique insight into alternate targets for these treatments and in part explain the disparate clinical responses patients exhibit.

levels at the site of infection and avoid the systemic toxicity associated with parenteral antibiotics. ${ }^{3-7}$ The first-line therapy used to treat chronic PA infections in $\mathrm{CF}$ is inhaled tobramycin powder/solution (TIP/S). ${ }^{3}$ Aggregate data collected from large clinical trials shows TIP/S improves lung function, quality of life, nutritional status, reduces pulmonary exacerbations (PEx) and even reduces mortality. ${ }^{3}$ 8-12 Despite the many clinical benefits afforded by these therapies, they do not result in sustained clearance of chronic infection but rather result in a variable decrease in $P$. aeruginosa bioburden $\left(1-2 \log _{10}\right.$ decrease Colony Forming Unit/g sputum). ${ }^{33}$ Moreover, patient outcomes have not reliably correlated with reductions in PA sputum density. ${ }^{14}$

The last two decades of research has revealed that the airways of individuals with CF are colonised by a diverse collection of organisms beyond the few identified by the clinical laboratory using traditional aerobic culture-this community has been termed the CF microbiome. Over time the CF microbiome changes as it evolves towards a climax structure. In fact, the diversity of the microbiome inversely correlates with age and positively correlates with lung function. ${ }^{15}$ Furthermore, this community experiences perturbations which are both associated with change in disease stage and acute parenteral 
antibiotic exposures. ${ }^{15-21}$ However, little is known about how the CF sputum microbiome may influence response to maintenance therapies. ${ }^{20-23}$ In the previous studies, our group examined the effects of the inhaled antibiotic aztreonam lysine (AZLI) on a cohort of treatment naïve patients, ${ }^{22}$ and during a single 28-day cycle of on/off AZLI. ${ }^{23}$ In both studies, we found that those who failed to respond to AZLI had microbiomes with a higher relative abundance (RA) of Staphylococcus spp-a Grampositive known to resist aztreonam. However, both studies were limited by their inclusion of a patient population with generally advanced lung disease given AZLI's role as a salvage agent. Accordingly, we developed a study focusing on the sputum microbiome of patients with $\mathrm{CF}$ and their response to the firstline antipseudomonal, tobramycin. We hypothesised that TIP/S has additional 'off-target' effects on the CF sputum microbiome and that the baseline microbiome prior to therapy initiation is associated with subsequent patient response.

\section{METHODS}

\section{Patients and sample collection}

The Calgary Adult Cystic Fibrosis Clinic has kept and maintained a frozen repository of sputum collected from CF adults since 1997. This biobank includes $>18000$ sputum samples collected from $>300$ individuals. Inclusion criteria for our study were chronic $P$. aeruginosa infection as defined by the Leeds criteria, ${ }^{24}$ receipt of $\mathrm{TIP} / \mathrm{S}$ for at least 1 year, and $\geq 1$ sputum sample collected within 18 months before and after the initiation of TIP/S. We excluded transplant recipients, and patients who had recent prior TIP/S (defined within 1 year of its index initiation). Similarly, we excluded samples collected from patients if they were collected within 30 days of a PEx and or/receipt of intravenous antibacterials.

Patient demographics including age, sex, CF and non-CF demographics, lung function (as measured by percent predicted forced expiratory volume in $1 \mathrm{~s}\left(\mathrm{ppFEV}_{1}\right)$ and percent predicted forced vital capacity (ppFVC)), nutritional status (as measured by body mass index), cultured classical-CF pathogens and medical therapies were collected for baseline samples (defined as first sample collected prior to treatment initiation).

\section{Definitions}

Definitions of lung disease stage were classified based on baseline $\mathrm{ppFEV}_{1}$; mild $\geq 70 \%$, moderate $\geq 40-<70 \%$, severe $<40 \%$. The primary outcome of interest was patient response. Our definition of response, selected prior to microbiome analysis, was achieving stable lung function measured by $\mathrm{ppEV}_{1}$ in the year following TIP/S initiation (no net decline, ie, zero change). This was chosen based on clinical trial data associated with TIP/S where improvements in lung function of $0 \%-8 \%$ were observed following treatment-and which generated two comparable groups. ${ }^{10}$ Median ppFEV per person was determined for each patient for the 18 months prior to and following TIP/S initiation. Change in $\mathrm{ppFEV}_{1}$ was calculated from pre vs post calculations and patients were classified as responders if they had no change or improvements in lung function. Additionally, a supplemental analysis was conducted to define response using changes in the slope of a linear regression in $\mathrm{ppFEV}_{1}$ pre-TIP versus post-TIP/S per patient. Exploratory analyses were performed on samples collected pre vs post TIP/S initiation. Samples associated with TIS and TIP were analysed in aggregate (as TIP was designed specifically to recapitulate the pharmacokinetics of TIS $^{15}$ ) and separately. Patients were analysed in aggregate and separately by exposure to TIP/S as treatment naïve, or not exposed to TIP/S for at least 5 years.

\section{Sputum DNA extraction, amplification and analysis}

Bacterial DNA was extracted from $300 \mu \mathrm{L}$ of homogenised sputum as previously described. ${ }^{22} 232526$ In brief, the V3 hypervariable region of the $16 \mathrm{~S}$ rRNA gene was amplified using reverse and forward barcoded primers on the Illumina MiSeq platform. ${ }^{25}$ qPCR was performed to determine the absolute abundance of $S$. aureus and the total bacterial load (defined as the number of $16 \mathrm{~S}$ rRNA copies/mL) in the sputum samples. ${ }^{27}$

Statistical analysis of operational taxonomic unit tables was carried out using phyloseq, ${ }^{28}$ vegan ${ }^{29}$ and DESeq $2^{30}$ packages in $\mathrm{R}$ (V.3.3.1). ${ }^{31}$ The Wilcoxon rank-sum test was used to describe changes in Shannon alpha-Diversity Indices (SDIs) in R. Community structures were assessed using Bray-Curtis (BC) betadiversity measures after proportionally normalising all samples by dividing read counts by the total number of reads. ${ }^{16}$ Permutational multivariate analyses of variance (PERMANOVAs) were used to analyse statistical differences in beta-diversity. Results were visualised using principal coordinate analysis plots. To account for repetitive sampling of patients (pre or post) we conducted a sensitivity analysis by setting constraints to permutations by patient ID. Restricted analyses were performed when assessing pre vs post samples to include the first presample (baseline-with the least prior exposure to TIP/S) and the last post sample (in order to observe the effects of prolonged TIP/S exposure). DESeq2 (test $=$ 'Wald' fitType $=$ parametric and Benjamin Hochberg multiple test correction) was used to determine genera that are differentially abundant, $\log _{2}$ of normalised reads were used to visualise data. Samples with an abundance value of 0 were changed to a value of 1 since $\log (0)$ is undefined and $\log (1)=0$.

\section{RESULTS}

\section{Cohort characteristics}

Forty-one patients contributing 151 sputum samples (median 4/ patient, IQR 3-4) representing before $(n=77)$ and after $(n=74)$ the initiation of TIP/S (figure 1) were analysed. Pre-TIP/S samples were collected at median day -91 (IQR -184 to -1 ) relative to initiation and samples collected after TIS/TIP at median day +146 (IQR 87-218). At baseline, 17 patients were naïve to $\mathrm{TIP} / \mathrm{S}$ having never been exposed, and 24 patients had remote exposure (a median 7 years free of TIP/S; IQR 2-8). Of the 41 patients, 26 initiated treatment with TIS and 15 TIP. TIS and TIP usage did not differ by response status or patient demographics including age or genetics. Patient demographics, chronic therapies and cultured pathogens at treatment initiation are presented in table 1 and online supplemental table S1. Patients were a median 30.4 years of age (IQR 24.2-35.2) and had a ppFEV 57\% (IQR 44-74) and ppFVC 87\% (IQR 72-93).

\section{Sputum microbiome composition by sex, response and patient ID}

A total of 6139490 sequences (median 32667 per sample (IQR 15351-63 128)) were generated from 151 sputum samples. Intrapatient and interpatient variability is observed in taxonomic summary plots for taxa present in $>20 \%$ of all samples (figure 1). When assessing all samples, we found patient ID explained $55 \%$ of the variation in beta-diversity (between sample diversity) as measured by BC dissimilarity (PERMANOVA, $\mathrm{R}^{2}=0.55, \mathrm{p}=0.001$ ). In addition, Pseudomonas 


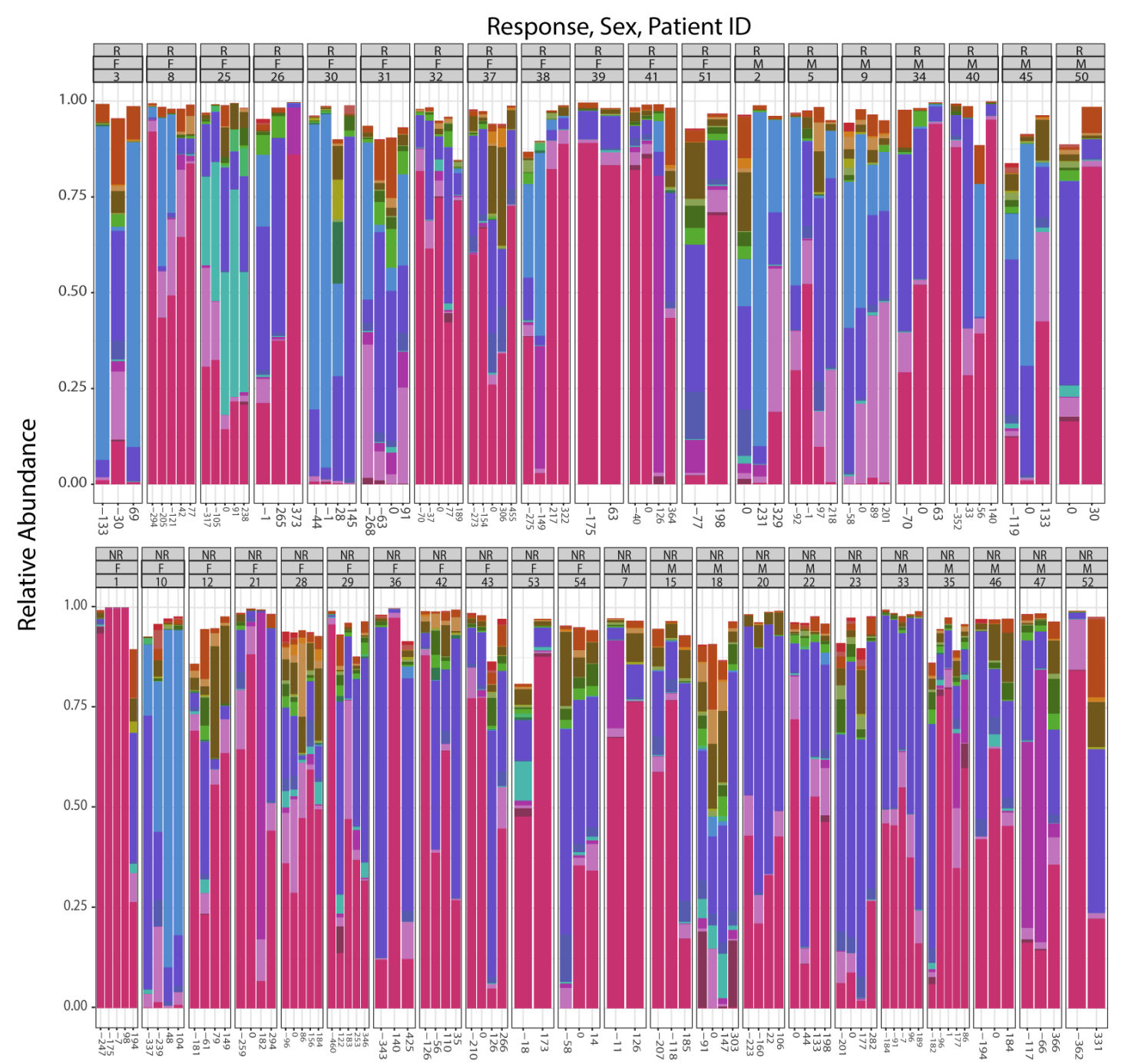

Days fom the Initiation of TIP/S

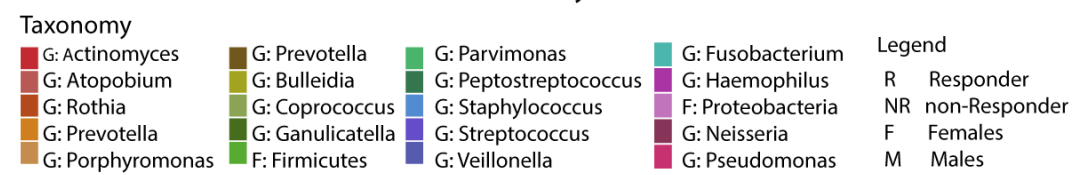

Figure 1 Microbiome as a function of days from the initiation of TIP/S. Taxonomic summaries for taxa present in $>20 \%$ of all samples ( $n=151$ ) for a cohort of 41 patients with chronic Pseudomonas aeruginosa infection. Days from the initiation of TIP/S are represented on the X-axis. Individual patient subplots are organised by response (R/NR), sex (F/M) and patient ID. NR, not responder; TIP/S, tobramycin inhalational powder/solution.

(the predominate pathogen) abundance explains $36 \%$ of the variance (PERMANOVA, $\left.\mathrm{R}^{2}=0.36, \mathrm{p}=0.001\right)$.

\section{Baseline sputum microbiome and its association with clinical response to TIP/S}

The primary goal for our study was to determine if baseline sputum microbiome features correlated with clinical response to $\mathrm{TIP} / \mathrm{S}$, which might suggest that microbiome may be used as a biomarker to predict response. Nineteen (46\%) patients met our a priori definition of response (R), exhibiting no decline in lung function in the year following initiation. The median $\mathrm{ppFEV}_{1}$ change in responders was $+2.5 \%$ (IQR $0.5 \%-6.5 \%$ ). Twenty-two patients (54\%) were defined as non-responders (NR) experiencing a median $\mathrm{ppFEV}_{1}$ drop of $-5.5 \%$ (IQR -3 to -9.5 ). When assessing baseline samples (collected closest to day 0 ) we found that treatment response explained $6 \%$ of the variation in beta-diversity (between sample diversity), as measured by BC dissimilarity between responders and nonresponders (PERMANOVA, $\mathrm{R}^{2}=0.061, \mathrm{p}=0.046^{*}$ ) (figure $2 \mathrm{~A}$ ). However, no differences were observed in SDI or other alphadiversity (within sample bacterial richness and evenness) metrics (figure 2B). Similar findings were observed for both BC and SDI metrics when we assessed baseline samples collected from patients exposed to TIS and TIP separately (data not shown).

Next, we sought to identify genus-level differences based on response. We observed non-responders to have $-5.28 \log _{2}$ lower RA of Staphylococcus spp compared with responders at baseline $(\mathrm{p}<0.001)$ (figure $2 \mathrm{C}$ and online supplemental table $\mathrm{S} 2)$. No other genera were significantly associated with treatment response although Pseudomonas spp trended towards a higher abundance in non-responders. As a sensitivity analysis, we assessed increasingly stringent definitions of response (online supplemental table S2). Definitions of response in our sensitivity analysis ranged from $-2.5 \%$ to $3 \%$ changes in lung function post 
Table 1 Cohort demographics and culture data for baseline samples collected prior to the initiation of chronic TIP/S

\begin{tabular}{|c|c|c|c|}
\hline \multirow[b]{2}{*}{ Characteristics of patient cohort } & \multicolumn{3}{|l|}{ Response* } \\
\hline & $\begin{array}{l}\text { Responder } \\
(n=19)(46 \%)\end{array}$ & $\begin{array}{l}\text { Non-Rt } \\
(n=22)(54 \%)\end{array}$ & $P$ value \\
\hline Female & $12(63)$ & $11(50)$ & 0.4 \\
\hline Median age, years (IQR) & $29.2(24.2-38.6)$ & $30.4(23.8-34.5)$ & 0.6 \\
\hline Median BMI $\left(\mathrm{Kg} / \mathrm{m}^{2}\right)$ & $22.1(20.4-23.6)$ & $21.1(19.3-22.4)$ & 0.3 \\
\hline \multicolumn{4}{|l|}{ Lung stage $\ddagger$} \\
\hline Mild & $5(26)$ & $10(45)$ & 0.4 \\
\hline Moderate & $9(47)$ & $8(36)$ & \\
\hline Severe & $5(26)$ & $4(18)$ & \\
\hline \multicolumn{4}{|l|}{ Genotype§ } \\
\hline$\Delta \mathrm{F} 508$ homozygous & $9(47)$ & $12(55)$ & 0.5 \\
\hline CF-related diabetes & $5(26)$ & $7(32)$ & 0.7 \\
\hline Liver disease & $6(32)$ & $3(14)$ & 0.2 \\
\hline Sinus disease & $5(26)$ & $6(27)$ & 0.9 \\
\hline \multicolumn{4}{|l|}{ Traditional cultured CF pathogens } \\
\hline Pseudomonas aeruginosa & $19(100)$ & $22(100)$ & $>0.99$ \\
\hline Methicillin susceptible Staphylococcus aureus & $6(32)$ & $2(9)$ & 0.07 \\
\hline Methicillin resistant $S$. aureus & 0 & 0 & NA \\
\hline Otherq & $2(11)$ & $3(14)$ & 0.8 \\
\hline
\end{tabular}

$\mathrm{X}^{2}$ tests were used to determine differences between dichotomous clinical variables. Wilcoxon rank-sum tests were conducted for analysis of continuous variables.

${ }^{*}$ Response was a priori defined as achieving stable lung function as measured by $\mathrm{FEV}_{1}$ per cent predicted in the year following TIP/S initiation (no net decline).

†Non-R=non-responder.

ҒDefinitions of lung stage based on forced expiratory volume in one second; mild $\geq 70 \%$, moderate $\geq 40$ to $<70 \%$, severe $<40 \%$.

$\S$ Genotypic data were unknown for one patient in the non-responder group.

ףOther bacterial species include Escherichia coli, Enterobacter cloacae, Serratia marcescens, Streptococcus Group C, Stenotrophomonas maltophilia.

$\mathrm{BMI}$, body mass index; CF, cystic fibrosis; NA, not applicable; TIP/S, tobramycin inhalational powder/solution.

treatment initiation. We chose negative values as a definition of response based on the median (IQR) decrease in lung function of non-responders (patients who fell below the 25 th percentile were considered as responders) and to generate a comparable number of patients in each group. A higher abundance of Staphylococcus spp continued to associate with response status in the majority of these alternate definitions for baseline pretreatment sputum. Furthermore, a linear regression comparing Staphylococcus RA as a function of change in lung function (following the initiation of TIP/S) trended towards a statistical correlation with a $\mathrm{R}^{2}$ of $0.09, \mathrm{p}=0.05$ ). To confirm the observed difference of Staphylococcus spp RA between responders and non-responders, we
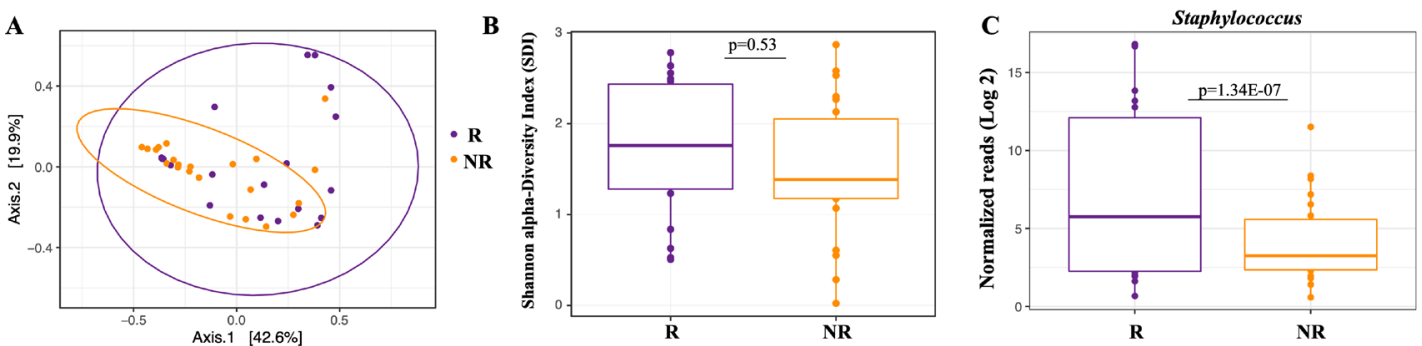

Figure 2 Baseline microbiome and associated response status for samples collected prior to TIP/S initiation. Responders were defined as patients who experienced no net decrease in lung function with TIP/S use. (A-C) Microbiome analyses of baseline samples only. (A) PcoA of Bray-Curtis dissimilarities showing community-wide differences between samples collected from $R(n=19)$ vs non- $R(n=22)\left(P E R M A N O V A, R^{2}=0.061, p=0.046^{*}\right)$. (B) Shannon alpha-Diversity Index (SDI) between $\mathrm{R}$ and non-R for baseline samples were not significantly different as demonstrated by Wilcoxon RANK sum test. (C) Log 2-fold of normalised reads reveal responders have a higher abundance of Staphylococcus as measured by a Wald's test using DESeq2. NR, not responder; PERMANOVA, permutational multivariate analysis of variance; TIP/S, tobramycin inhalational powder/solution. 
A

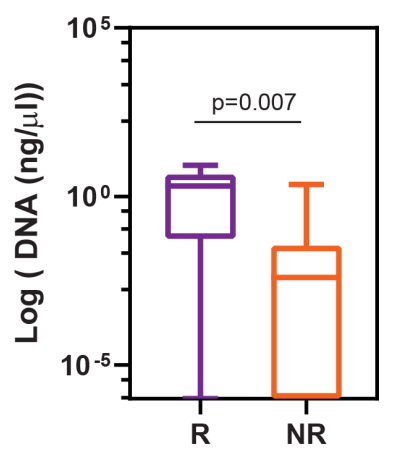

B

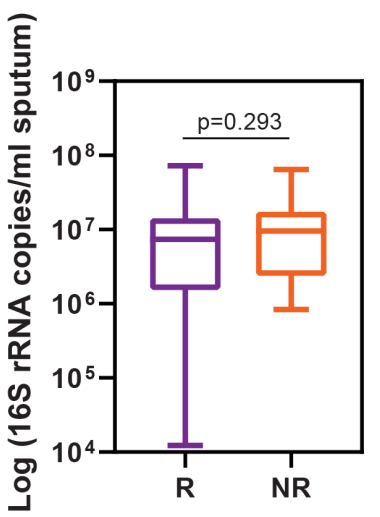

Figure 3 Response status as a function of total genomic DNA. (A) Absolute abundance ( $\log 10)$ of Staphylococcus aureus in $400 \mathrm{ng}$ of extracted DNA (bacterial and host) from sputum calculated by qPCR with primers to $r n p B$ (Ba04646259_s1; thermo Fisher scientific). qPCR for $S$. aureus detection from $R(n=16)$ vs non- $R(n=20)$ demonstrated Staphylococcus DNA absolute abundance was greater in responders (as determined by Mann-Whitney $U$ test). Five samples were not included in the analysis as the concentration of total genomic DNA was too low (3 $R, 2$ NR). (B) Comparison of total sputum bacterial load as determined by 165 rDNA was not different in $R(n=19)$ vs non- $R(n=22)$. NR, not responder; $q P C R$, quantitative $P C R$.

quantified the absolute abundance of $S$. aureus present in a given amount of DNA (400 ng total extracted DNA) using qPCR. We observed non-responders to have lower abundance of $S$. aureus compared with responders $(\mathrm{p}=0.007)$ (figure $3 \mathrm{~A})$. Additionally, no differences were observed in the total bacterial load between responders and non-responders at baseline (figure $3 \mathrm{~B}$ ).

Moreover, and perhaps surprisingly, a lower abundance of Pseudomonas spp was associated with response to TIP/S in all but our a priori response definition (online supplemental table S2). Other organisms including Mogibacterium spp and Scardovia spp were also associated with response although were present in low base mean reads and may be less biologically relevant. With increasingly stringent definitions of response, non-responders trended towards significantly decreased alpha-diversity compared with responders (online supplemental table S2). RA of Staphylococcus spp also outperformed SDI in receiver operating characteristic (ROC) curve analysis (online supplemental figure S1). In addition, a supplemental analysis was conducted to define response using the slope of a linear regression. Although no differences were observed in alpha-diversity or beta-diversity, responders had a higher abundance of Staphylococcus at baseline compared with non-responders further supporting our findings.

\section{Changes in the sputum microbiome preinhaled versus postinhaled tobramycin treatment}

Our next objective was to determine if changes in long-term microbial community structure associated with the initiation of TIP/S. No significant differences in sputum microbiota (BC) were identified when comparing all samples collected prior $(n=77)$ to and post $(n=74)$ initiation of TIP/S (PERMANOVA, $\left.\mathrm{R}^{2}=0.0033, \mathrm{p}=0.84\right)$. Similar findings were observed when analyses were performed to account for multiple samples/patient and constrained by patient ID (PERMANOVA, $\mathrm{R}^{2}=0.0033, \mathrm{p}=0.85$ ) (online supplemental figure S2). No differences were observed in SDI for samples collected preinitiation versus postinitiation of TIP/S (online supplemental figure S2). A secondary analysis

using a generalised estimating equation was conducted to determine if baseline SDI and Staphylococcus RA influenced changes in the SDI before and after TIP/S initiation, however, no significant differences were observed (data not shown). These findings were sustained when restricted analyses were performed assessing SDI using samples collected at baseline pre $(n=41)$ vs last post $(n=41)$ initiation of TIP/S (Wilcoxon, $p=0.54)$. When we assessed the first pre and last post samples we found a -3.8 $\log _{2}$ fold decrease in Parvimonas spp $(\mathrm{p}=0.0001)$ although the base mean reads were low and organisms belonging to this genus may be less biologically relevant (data not shown). Similar findings were observed when TIS and TIP exposed patients were assessed separately. No significant differences were observed in either BC or SDI indices pre vs post initiation of therapy (data not shown).

Next, we assessed microbiome changes in baseline pre versus the last available sample collected separately for each of responders and non-responders. No significant differences were noted for beta-diversity of responders pre-TIP vs post-TIP/S (PERMANOVA, $\mathrm{R}^{2}=0.04, \mathrm{p}=0.17$ ). However, responders had a significant decrease in SDI in the last available postsample compared with baseline $(p=0.046)$. Furthermore, no taxa were differentially abundant for responders pre-TIP vs post-TIP/S. Non-responders showed no significant difference in BC betadiversity pre-TIP vs post-TIP/S (PERMANOVA, $\mathrm{R}^{2}=0.026$, $\mathrm{p}=0.33)$. Similar findings were observed for SDI where no differences were noted in non-responders pre-TIP vs post-TIP/S $(\mathrm{p}=0.20)$. Notably, however, non-responders had a $-3.1 \mathrm{log}$ 2 -fold decrease Pseudomonas spp $(\mathrm{p}<0.001)$ and $-2.8 \log 2$-fold decrease in Leptotrichia spp $(\mathrm{p}=0.027)$ post-TIP/S initiation.

As the sputum microbiome of individuals with mild lung disease $\left(\geq 70 \% \mathrm{FEV}_{1}\right.$ ) generally trends towards higher SDI and is potentially more susceptible to deviations with medical interventions, we did a subgroup analysis where we assessed only those 15 patients with mild lung disease. No differences were observed in baseline pretherapy and last post-therapy samples collected from patients with mild lung disease in BC beta-diversity, SDI, and no taxa were differentially abundant (data not shown).

Finally, we performed a subgroup analysis in those patients who were TIP/TIS naïve as well as in those who had not had exposure in $\geq 5$ years (in order to eliminate any potential confounding from past prior exposures) and results were similar in diversity measures in both of these subgroups. No significant differences were observed in samples collect pre versus last post for BC betadiversity or SDI in either the naïve group or the group with no $\mathrm{TIP} / \mathrm{S}$ exposure in $\geq 5$ years. Similarly, no taxa were differentially abundant in samples collect pre versus last post in patients that were naïve to TIP/S. However, patients who had not had TIP/S exposure in $\geq 5$ years showed significant decrease in Parvimonas and Mogibacterium in the samples collected pre versus last post (data not shown).

\section{DISCUSSION}

The CF sputum microbiome consists of a diverse group of organisms. The role of this community in health and disease remains to be fully determined, but already it has been associated with age, sex, lung disease stage and the occurrence of PEx. ${ }^{151827}$ Our group previously assessed changes in the sputum microbiome following the initiation of $\mathrm{AZLI}^{22}$ and during a single cycle of AZLI therapy. ${ }^{23}$ Our findings suggested that the sputum microbiome is relatively resistant to AZLI although intrapatient differences were observed 'on' vs 'off' treatment. In both of these studies non-responders had a higher abundance 
of Staphylococcus spp-an organism intrinsically resistant to aztreonam. As $S$. aureus has now become the most prevalent CF pathogen-these results proved intriguing and suggested that baseline microbiota may influence response to therapiespotentially serving as a biomarker to enable treatment personalisation. ${ }^{32}$ However, both studies were limited with the inclusion of patients with more advanced lung disease (given AZLI's use as a salvage agent in Canada) - thereby having a less diverse microbiome-potentially less susceptible to perturbation. To address these limitations, here we completed a similar study to understand the effect and association of TIP/S-the first-line treatment for chronic $P$. aeruginosa infection in $\mathrm{CF}-$ on $\mathrm{CF}$ sputum microbiome.

Interestingly, patients who responded to TIP/S had baseline differences in sputum bacterial community composition compared with non-responders as determined by BC beta-diversity analyses. Such findings may support a role for sputum microbiota to be used as a biomarker for personalisation of therapies. In particular, these differences were characterised by a higher abundance of Staphylococcus in baseline samples collected from responders compared with non-responders. This is an intriguing observation, distinctly contrasting what our group has observed with the monobactam AZLI. However, tobramycin-an aminoglycoside-functions by interfering with bacterial protein synthesis by binding the 30 S-ribosomal subunit. ${ }^{33}$ Like aztreonam, tobramycin's spectrum of activity is generally confined to aerobic Gram-negative organisms-with the very notable exception of Staphylococci. ${ }^{34}$ Indeed, both methicillin-sensitive $S$. aureus (MSSA) and methicillin-resistant $S$. aureus are generally susceptible to tobramycin. ${ }^{35} 36$ When we assessed 57 MSSA isolates collected from 30 individuals from this cohort derived from a concomitant pathogen biobank collected a median 44 days (IQR -104 to 337 ) from TIP/S initiation we determined the median tobramycin minimal inhibitory concentration of these isolates was $0.75 \mu \mathrm{g} / \mathrm{mL}$ (IQR0.5-1.0)—well below the levels achieved with TIP/S 34 (data not shown). Given the increasing prevalence of MSSA (infecting 53.6\% of Canadians in 2017), having recently surpassed $P$. aeruginosa (infecting $40.2 \%$ ) as the most prevalent pathogen-new treatment strategies targeting this organism are required. ${ }^{32}$ In contrast, responders had lower RA of Pseudomonas, the intended target of TIP/S, compared with non-responders. Together, these data suggest that the current practice of only using cycled TIP/S in $P$. aeruginosa chronically infected patients may be inappropriately exclusionary. Future clinical trials assessing the potential clinical benefit of TIP/S for patients with CF with chronic $S$. aureus infection may thus be in order. As this drug is already licensed, and care providers are very familiar with its use-a prospective randomised control trial demonstrating clinical benefit could immediately result in patient access. Indeed, 'off -label' $\mathrm{TIP} / \mathrm{S}$ use in $P$. aeruginosa uninfected individuals may already be common in PEx prone individuals.

Furthermore, responders trended towards higher SDI compared with non-responders at baseline. Concomitant with these findings, responders demonstrated a lower Pseudomonas and higher Staphylococcus absolute and RA in contrast to nonresponders suggesting a propensity towards response to treatment may be linked to baseline community composition. All of these findings suggest that our use of tobramycin exclusively in those with chronic $P$. aeruginosa infection may in fact be too limited and those with earlier disease (and those without chronic $P$. aeruginosa infection) may have the potential for benefit of TIP/S therapies. When we conducted a ROC curve analysis to evaluate how accurately RA of Staphylococcus and
SDI predict response-we found RA of Staphylococcus was superior to SDI.

A recently published manuscript by Nelson et al follows a cohort of non-naïve patients through a single TIP cycle, complements and supports the findings herein as they too have observed the greatest short-term impacts of TIP were on noncanonical sputum constituents and its effects independent of the targeted pathogen. ${ }^{38}$ In addition to differences in study design and tobramycin exposure, our cohorts have different characteristics including microbial community make-up with $P$. aeruginosa being more abundant in our cohort. Whereas, Nelson et al, observed differences in community structure at specifically selected time points where TIP usage was controlled, we did not observe consistent and significant long-term changes in the microbiome before and after TIP/S. ${ }^{17}{ }^{38}$ Indeed, these findings mirror our prior observations with AZLI. The most important limitation to our observation is our inability to reliably discern whether patients in our cohort were 'on' or 'off' TIP/S 28-day cycle in our biobanked samples (historically this clinical information was not documented).

We acknowledge a number of other limitations to our work. Our study focused exclusively on adult patients. We determined sputum samples with a higher abundance of Staphylococcus spp were more likely to occur in responders to TIP/S. While we are unable to confirm that these reads were specific to $S$. aureus as opposed to another Staphylococcal species (difficult to discern from 16S data)-we confirmed this with $S$. aureusspecific qPCR. Furthermore, $S$. aureus was cultivated in real time from many specimens. Using culture-independent technologies meant, we were unable to determine whether patients in our cohort had $S$. aureus small colony variants and if this was associated with response-relevant as this phenotype has previously associated with exaggerated lung function decline. ${ }^{39}$ While our study focused exclusively on inhaled tobramycin, it is likely that most individuals in our cohort had been remotely exposed to parenteral formulations of tobramycin during PEx-and these may have transiently influenced the microbiome although we expect these to be transient shifts, long since resolved. ${ }^{17}$ Furthermore, in this retrospective study, samples were collected at clinical visits irrespective of TIP/S initiation which is a limitation of our study design.

As the management of CF involves multiple complementary therapies all started at different times-each of which may impact the sputum microbiome-attributing changes to one specific therapy is exceedingly complex. Furthermore, previous studies have shown regional diversity in microbiome of lungs, and significant potential for oropharyngeal contamination which may be not have been appreciated based on our study design using expectorated sputum. ${ }^{40}$ However, invasive procedures such as bronchoalveolar lavage pose patient risk and are not a feasible for routine screening. ${ }^{4041}$ While we focused solely on microbial community composition, future prospective studies could assess functional differences in the metagenome (as done by Nelson et $a l$,) and metatranscriptome-unfortunately impossible for biobanked samples such as ours. Additionally, culture enriched metagenomics may be implemented to gain a better understanding of microbial communities specifically low abundant organisms. ${ }^{42}$ In the design of future clinical trials, it will be key to biobank samples including urine, serum, sputum, faeces and cultured-bacteria to better understand intricate host-microbial changes in response to different therapeutics. 


\section{CONCLUSIONS}

Our study confirms previous findings suggesting that the longterm CF sputum microbiome is relatively resistant to change following initiation of inhaled antibiotics. Intriguingly, differences in baseline microbiome were observed between responders and non-responders suggesting a potential for sputum to be used as a precision medicine tool to predict patient response to therapies. Interestingly, responders had a higher abundance of $S$. aureus at baseline suggesting that this organism may play a role in response to TIP/S although this warrants further investigation.

Acknowledgements The authors gratefully acknowledge the participation of patients and staff at the CACFC. The authors further acknowledge Calgary Laboratory Services and Alberta Precision Laboratories for assistance in collecting and storing samples.

Contributors All authors meet requirements for authorship having contributed significantly to the project. AH, CT and MDP designed the study. AH, CT and MDP collected clinical data. HR, RS and MDP collected patient samples. AH performed experiments and was responsible for data analysis. Sample processing and statistica analyses were performed by $A H, N A$, ILL, LR, DS, RS, MS and MDP. AH wrote the initial draft of the manuscript, and all authors contributed to its revision. MDP supervised the study and serves as guarantor of the work.

Funding This work was supported by a grant to MDP from CIHR (364568).

Competing interests MDP, HR and MS have received research support from CF Canada, CIHR and Gilead.

Patient consent for publication Not required.

Ethics approval All patients provide prospective consent for the collection and storage of sputum samples for research purposes as approved by the Calgary Health Region Ethics Board (REB15-0854).

Provenance and peer review Not commissioned; externally peer reviewed.

Data availability statement Data from this project are available at; https://www. ncbi.nlm.nih.gov/bioproject/662963

\section{REFERENCES}

1 Lipuma JJ. The changing microbial epidemiology in cystic fibrosis. Clin Microbiol Rev 2010;23:299-323.

2 Mogayzel PJ, Naureckas ET, Robinson KA, et al. Cystic fibrosis pulmonary guidelines. chronic medications for maintenance of lung health. Am J Respir Crit Care Med 2013;187:680-9.

3 Ramsey BW, Pepe MS, Quan JM, et al. Intermittent administration of inhaled tobramycin in patients with cystic fibrosis. cystic fibrosis inhaled tobramycin Study Group. N Engl J Med 1999:340:23-30.

4 LiPuma JJ. Microbiological and immunologic considerations with aerosolized drug delivery. Chest 2001;120:118S-23.

5 Touw DJ, Knox AJ, Smyth A. Population pharmacokinetics of tobramycin administered thrice daily and once daily in children and adults with cystic fibrosis. J Cyst Fibros 2007;6:327-33

6 Prayle A, Smyth AR. Aminoglycoside use in cystic fibrosis: therapeutic strategies and toxicity. Curr Opin Pulm Med 2010;16:604-10.

7 Smith AL. Inhaled antibiotic therapy: what drug? what dose? what regimen? what formulation? J Cyst Fibros 2002;1:189-93.

8 Gibson RL, Emerson J, McNamara S, et al. Significant microbiological effect of inhaled tobramycin in young children with cystic fibrosis. Am J Respir Crit Care Med 2003;167:841-9.

9 Ramsey BW, Dorkin HL, Eisenberg JD, et al. Efficacy of aerosolized tobramycin in patients with cystic fibrosis. N Engl J Med 1993;328:1740-6.

10 Konstan MW, Flume PA, Kappler M, et al. Safety, efficacy and convenience of tobramycin inhalation powder in cystic fibrosis patients: the EAGER trial. J Cyst Fibros 2011;10:54-61.

11 Konstan MW, Geller DE, Minić P, et al. Tobramycin inhalation powder for P. aeruginosa infection in cystic fibrosis: the evolve trial. Pediatr Pulmonol 2011;46:230-8.

12 Geller DE, Konstan MW, Smith J, et al. Novel tobramycin inhalation powder in cystic fibrosis subjects: pharmacokinetics and safety. Pediatr Pulmonol 2007:42:307-13.
13 Retsch-Bogart GZ, Quittner AL, Gibson RL, et al. Efficacy and safety of inhaled aztreonam lysine for airway Pseudomonas in cystic fibrosis. Chest 2009; 135:1223-32.

14 Gibson RL, Retsch-Bogart GZ, Oermann C, et al. Microbiology, safety, and pharmacokinetics of aztreonam lysinate for inhalation in patients with cystic fibrosis. Pediatr Pulmonol 2006:41:656-65.

15 Coburn B, Wang PW, Diaz Caballero J, et al. Lung microbiota across age and disease stage in cystic fibrosis. Sci Rep 2015;5:10241.

16 Carmody LA, Zhao J, Kalikin LM, et al. The daily dynamics of cystic fibrosis airway microbiota during clinical stability and at exacerbation. Microbiome 2015;3:12.

17 Smith DJ, Badrick AC, Zakrzewski M, et al. Pyrosequencing reveals transient cystic fibrosis lung microbiome changes with intravenous antibiotics. Eur Respir J 2014:44:922-30.

18 Whelan FJ, Heirali AA, Rossi L, et al. Longitudinal sampling of the lung microbiota in individuals with cystic fibrosis. PLoS One 2017;12:e0172811.

19 Layeghifard M, Li H, Wang PW, et al. Microbiome networks and change-point analysis reveal key community changes associated with cystic fibrosis pulmonary exacerbations. NPJ Biofilms Microbiomes 2019;5:4.

20 Caverly LJ, Lu J, Carmody LA, et al. Measures of cystic fibrosis airway microbiota during periods of clinical stability. Ann Am Thorac Soc 2019;16:1534-42.

21 Price $\mathrm{KE}$, Hampton $\mathrm{TH}$, Gifford $\mathrm{AH}$, et al. Unique microbial communities persist in individual cystic fibrosis patients throughout a clinical exacerbation. Microbiome 2013;1:27.

22 Heirali AA, Workentine ML, Acosta N, et al. The effects of inhaled aztreonam on the cystic fibrosis lung microbiome. Microbiome 2017;5:51.

23 Heirali AA, Acosta N, Storey DG, et al. The effects of cycled inhaled aztreonam on the cystic fibrosis (CF) lung microbiome. J Cyst Fibros 2019;18:829-37.

24 Lee TWR, Brownlee KG, Conway SP, et al. Evaluation of a new definition fo chronic Pseudomonas aeruginosa infection in cystic fibrosis patients. J Cyst Fibros 2003;2:29-34.

25 Bartram AK, Lynch MDJ, Stearns JC, et al. Generation of multimillion-sequence $16 \mathrm{~S}$ rRNA gene libraries from complex microbial communities by assembling paired-end Illumina reads. App/ Environ Microbiol 2011:77:3846-52.

26 Heirali A, McKeon S, Purighalla S, et al. Assessment of the microbial constituents of the home environment of individuals with cystic fibrosis (CF) and their association with lower airways infections. PLoS One 2016;11:e0148534

27 Zhao J, Schloss PD, Kalikin LM, et al. Decade-Long bacterial community dynamics in cystic fibrosis airways. Proc Natl Acad Sci U S A 2012;109:5809-14.

28 McMurdie PJ, Holmes S. phyloseq: an R package for reproducible interactive analysis and graphics of microbiome census data. PLoS One 2013;8:e61217.

29 Oksanen J. Multivariate Analysis of Ecological Communities in $R$ : vegan tutorial, 2011: 922.

30 Love MI, Huber W, Anders S. Moderated estimation of fold change and dispersion for RNA-Seq data with DESeq2. Genome Biol 2014;15:550.

31 Loo MPJVder. Learning RStudio for R statistical computing, 2012.

32 Cystic Fibrosis Canada. 2017/2018 annual report, 2017.

33 Lynch SR, Puglisi JD. Structural origins of aminoglycoside specificity for prokaryotic ribosomes. J Mol Biol 2001:306:1037-58.

34 Lam J, Vaughan S, Parkins MD. Tobramycin inhalation powder (tip): an efficient treatment strategy for the management of chronic Pseudomonas aeruginosa infection in cystic fibrosis. Clin Med Insights Circ Respir Pulm Med 2013;7:CCRPM.S10592-77.

35 Simon VK, Mösinger EU, Malerczy V. Pharmacokinetic studies of tobramycin and gentamicin. Antimicrob Agents Chemother 1973;3:445-50.

36 Dienstag J, Neu HC. In vitro studies of tobramycin, an aminoglycoside antibiotic. Antimicrob Agents Chemother 1972:1:41-5.

37 Somayaji R, Parkins MD. Tobramycin inhalation powder: an efficient and efficacious therapy for the treatment of Pseudomonas aeruginosa infection in cystic fibrosis. Ther Deliv 2015;6:121-37

38 Nelson MT, Wolter DJ, Eng A, et al. Maintenance tobramycin primarily affects untargeted bacteria in the CF sputum microbiome. Thorax 2020;75:780-90.

39 Wolter DJ, Emerson JC, McNamara S, et al. Staphylococcus aureus small-colony variants are independently associated with worse lung disease in children with cystic fibrosis. Clin Infect Dis 2013;57:384-91.

40 Boutin S, Graeber SY, Weitnauer M, et al. Comparison of microbiomes from different niches of upper and lower airways in children and adolescents with cystic fibrosis. PLoS One 2015:10:e0116029.

41 Tunney MM, Field TR, Moriarty TF, et al. Detection of anaerobic bacteria in high numbers in sputum from patients with cystic fibrosis. Am J Respir Crit Care Med 2008;177:995-1001.

42 Whelan FJ, Waddell B, Syed SA, et al. Culture-enriched metagenomic sequencing enables in-depth profiling of the cystic fibrosis lung microbiota. Nat Microbio 2020;5:379-90. 